\title{
GENDERED WORDS IN CANADIAN ENGINEERING RECRUITMENT DOCUMENTS
}

\author{
Agnes G. d'Entremont, $P h D^{1}$, Kerry Greer, $P h D^{2}$, and Katherine A. Lyon, $M A^{2}$ \\ ${ }^{1}$ Department of Mechanical Engineering and ${ }^{2}$ Department of Sociology, University of British Columbia \\ agnes.dentremont@mech.ubc.ca, kerry.greer@ubc.ca
}

\begin{abstract}
Gender imbalance exists in nearly every undergraduate engineering department in Canada. There is evidence that subtle gendered wording may influence perceptions of women's fit in a field. In this study, we applied a content analysis approach to evaluating the presence of gendered words in engineering undergraduate recruitment materials and compared these results to enrolment data and other factors for 18 English-language Canadian engineering schools. To our knowledge, this is the first evaluation of gendered wording in recruitment materials as a factor in the enrolment of women in engineering. We found that the use of feminine wording in recruitment materials has a weak, negative correlation with enrolment of women students, but a positive correlation with percentage of female faculty members. Our data point to the need for further analysis of recruitment material and with attention focused on the performance of gender in this material, and how it and other factors may influence enrolment.
\end{abstract}

Keywords: Gender, recruitment, language, content analysis

\section{INTRODUCTION}

Gender imbalance exists in nearly every undergraduate engineering faculty in Canada [1], despite concerted efforts to attract women to engineering [2]. Overall Canadian female enrolment in undergraduate engineering has remained close to or under $20 \%$ for the past 15 years [1]. While women's entrance into engineering has stagnated, over this same time period women's enrolment in university has reached (and exceeded) parity with men in 1987 [3]. Research in the U.K. shows that recruitment of women into a diverse range of scientific fields has been achieved and that parity has been attained, but that the proportion of women in Engineering continues to lag, despite aggressive efforts [4].

While there is some evidence of higher attrition of female students in engineering compared to their male peers [5], much of the discrepancy starts before students even set foot on campus [6], with lower numbers of women applying for engineering programs. Supply side theories posit that one explanation for the low number of women in Engineering is that women recognize the high demands of the field, and drop out [7]. Unlike biology or psychology, two fields where women have attained gender parity, this theoretical perspective asserts that women get the message that Engineering is less conducive to achieving other life goals, such as having and rearing a child. The National Graduate Survey of Canadians [8] suggests that students select degree programs based on future employment opportunities and their personal interests. Rather than being driven away from engineering because of future expectations related to reproduction, instead it seems more likely that engineering produces a culture that is perceived by potential women students as unfeminine. Acker [9] argues that organizations can embody a distinctly male orientation, which is communicated in part through gendered images and symbols within language.

This research considers this possibility by examining the content of recruitment materials produced by engineering programs. Evidence in the literature suggests that the use of words associated as masculine and feminine in job advertisements may influence a woman's decision to apply for a particular job [10]. It should be noted that these are not explicitly gendered words, such as male pronouns (e.g. he, him) or gendered job titles (e.g. serviceman), but rather descriptive words that are associated implicitly with male or female gender (e.g. nurture, aggressive). Similar evidence of broad-based implicit bias has been demonstrated through associations of women/female descriptors with words related to the humanities and $\mathrm{men} / \mathrm{male}$ descriptors with words related to the sciences [11] (see also Harvard Implicit Bias test, https://implicit.harvard.edu).

Gaucher et al. proposed that "gendered wording may emerge within job advertisements as a subtle mechanism of maintaining gender inequality by keeping women out of male-dominated jobs" [10], and there is evidence of the effect of these words on perceptions of percentage of women and women's "fit" in an occupation [10]. It is reasonable to imagine that the same may be true for entry 
into the profession of engineering at the undergraduate recruitment stage.

To determine the gendered orientation of engineering programs we examined the website pages of a sample of Canadian engineering universities, computing the frequency for a list of gendered words compiled by Gaucher et al. We supplemented these data with information about the gendered composition of the faculty and students in engineering at each university and analyzed these correlations to determine whether schools with greater feminine words saw an increase in women enrolments. We found that there is a weak negative association between the proportion of words that are feminine and the proportion of women enrolled. We also analyzed the data to determine whether there are differences by discipline.

\section{METHODOLOGY}

\subsection{Overview of Methods}

We performed content analysis of collected, publically available material from English-language Canadian engineering schools, to evaluate the presence and frequency of gendered words.

The list of gendered words used in this analysis was compiled by Gaucher et al. from studies looking at job advertisements. They examined job advertisements in 11 fields that were traditionally male-dominated and traditionally female-dominated for gendered words. They found that male-dominated jobs tended to have more masculine words than female-dominated jobs, however no significant difference was found in the number of feminine words. They replicated this finding in university co-op job advertisements (engineering and arts). Following this analysis, they applied their findings to create job advertisements that included the masculine and feminine words, and research subjects reported lower estimates of women in a profession when more masculine words were used, regardless of the profession in the ad or the gender of the research subject. Female research subjects also rated masculine-worded jobs as less appealing and anticipated not fitting in as well, even when the job ad was for a field not perceived as dominated by one gender (e.g. real estate).

The idea that subtle changes in wording may influence women's perception of a field may be important in increasing the number of women enrolling in engineering undergraduate programs. To examine this idea, we collected materials from Canadian engineering programs aimed at prospective students in order to examine them for gendered wording and compare those results to actual enrolments in both schools and in disciplines within engineering.

\subsection{Institutions and Materials}

From the population of all 38 accredited, Canadian, English-language engineering schools, we selected a sample of 18 schools to be included in the analysis. Schools were selected to include a wide range of enrolment size and percentage of women undergraduates enrolled (based on Engineers Canada data [1]), as well as covering all provinces. The range of enrolment also tended to provide a range of number of engineering disciplines per school (see Table 1).

Web-based material aimed at prospective students (webpages, text, images, videos) was collected for each school over a three-month period and analyzed for content. Information that described the program, careers in the discipline, support for students provided by the engineering school or program, and other material that expressed why a student should enroll in that school or program was included. Material that was administrative in nature (for example, information on how to apply into the program, or a list of course numbers and names) was excluded. Information about topics such as fundraising and donors, faculty and staff (e.g. biographies), bursaries, strategic planning, graduate programs, non-engineering programs within an engineering faculty (e.g. architecture), and resources available to any undergraduate in the institution (including those outside of engineering) was also excluded. Information on research was generally excluded (unless it was aimed at an undergraduate audience). Number of documents collected and included for each school varied greatly, depending on how information was divided between webpages, the number of program-specific sub-sites, the amount of administrative versus promotional content, and the original amount of web content put online by each school.

Each occurrence of a gendered word was treated as a single observation, and was tied to both university level and disciplinary level data. We used Engineering Canada data for the 2013-2014 school year to obtain the percent of female students enrolled in both the school and in each engineering discipline (e.g. Mechanical, Chemical), and the percent of women on the faculty [1]. New programs that started enrolling in 2014-2015 or will start in the future were excluded due to a lack of enrolment data for those programs.

\subsection{Gendered Words}

Gendered words were obtained from the work of Gaucher et al., where they evaluated job advertisements for a number of gendered occupations (including engineering) for gendered words [10]. 41 masculine and 39 feminine words were examined in this study. See Appendix A for a list of the occupations and a complete list of words determined as part of their study. 
One coder searched all the collected engineering school text-based documents for use of gendered words [10]. In cases where the word was used in a technical way to describe a device, principle, or process (e.g. "autonomous" robot, "force" as a physical quantity), the instance was excluded. In cases where the word described a person, group of people, the engineering community or climate (e.g. students work "autonomously" on labs, or engineering being a "force for good" in the world), the word was included. The total number of words in the materials analyzed from each university website was also calculated, and this was used to calculate the percent of words that were feminine and percent of words that were masculine.

Each document was also coded for school and discipline (if any), and each program or discipline was categorized using the categories provided by Engineers Canada (see Appendix $\mathrm{C}$ of [1]), with an additional category created for general or faculty-wide material.

\subsection{Statistical Analysis}

Two sets of analysis were conducted. The first examined school-level data. We calculated the correlations between the fraction of gendered words, fraction of female faculty, and the fraction of women students enrolled in each school. We then performed an Ordinary Least Squares regression to determine the relationships between gendered words (dependent variable), female enrolment and female faculty, while controlling for the total words on the website.

A second set of analysis was conducted looking at discipline-level data. This analysis was more restricted, as we did not have data on faculty members by discipline, but were able to calculate the correlation between the fraction of gendered words by discipline, and the fraction of women students enrolled in each discipline. We restricted this analysis to only defined disciplines, dropping the observations of gendered words on general web pages and in common year programs. Using this restricted dataset, we computed Pearson's correlation coefficients for fraction of gendered words and fraction of women students enrolled in each discipline.

All statistical analyses were performed using STATA (StataCorp, College Station, Texas).

Table 1: Universities included in analysis, and their characteristics from Engineers Canada [1]

\begin{tabular}{|c|c|c|c|c|c|}
\hline University & Province & $\begin{array}{l}\text { No. of Disciplines (Eng. } \\
\text { Can. categories, } \\
\text { excluding common } \\
\text { year(s)) }\end{array}$ & $\begin{array}{l}\text { Total Enrolment } \\
(2013)\end{array}$ & $\begin{array}{l}\text { Proportion } \\
\text { of Women } \\
\text { Enrolled } \\
(2013) \\
\end{array}$ & $\begin{array}{l}\text { Proportion of } \\
\text { Tenured/Tenure } \\
\text { Track Female } \\
\text { Faculty (2013) } \\
\end{array}$ \\
\hline Alberta & $A B$ & 9 & 4145 & $20 \%$ & $9 \%$ \\
\hline $\mathrm{BCIT}$ & $\mathrm{BC}$ & 2 & 487 & $9 \%$ & $12 \%$ \\
\hline Calgary & $A B$ & 7 & 3190 & $24 \%$ & $15 \%$ \\
\hline Carleton & $\mathrm{ON}$ & 9 & 3228 & $14 \%$ & $10 \%$ \\
\hline Concordia & QC & 6 & 3090 & $20 \%$ & $16 \%$ \\
\hline Conestoga & ON & 1 & 98 & $5 \%$ & $10 \%$ \\
\hline Dalhousie & NS & 10 & 1342 & $20 \%$ & $15 \%$ \\
\hline Manitoba & $\mathrm{MB}$ & 5 & 1412 & $18 \%$ & $12 \%$ \\
\hline McGill & QC & 8 & 2649 & $23 \%$ & $11 \%$ \\
\hline Memorial & $\mathrm{NL}$ & 5 & 939 & $26 \%$ & $13 \%$ \\
\hline Regina & SK & 5 & 1157 & $23 \%$ & $17 \%$ \\
\hline SFU & $\mathrm{BC}$ & 2 & 1119 & $14 \%$ & $15 \%$ \\
\hline StFX & NS & 0 (Common year(s) only) & 90 & $29 \%$ & $0 \%$ \\
\hline UBCO & $\mathrm{BC}$ & 3 & 262 & $13 \%$ & $13 \%$ \\
\hline UBCV & $\mathrm{BC}$ & 12 & 3699 & $21 \%$ & $10 \%$ \\
\hline UNB & NB & 9 & 1671 & $15 \%$ & $13 \%$ \\
\hline UPEI & $\mathrm{PE}$ & 0 (Common year(s) only) & 126 & $14 \%$ & $25 \%$ \\
\hline Waterloo & $\mathrm{ON}$ & 9 & 5185 & $19 \%$ & $14 \%$ \\
\hline Summary & 10 Provinces & 0-12 Disciplines & 90-5185 Enrolled & Mean $18 \%$ & Mean $13 \%$ \\
\hline
\end{tabular}

\section{RESULTS}

We found 38 unique gendered words in the web materials collected, 21 masculine and 17 feminine words.

When we examined the number of instances of each gendered word over all schools, the top five were: Lead* (m, 90 instances), Support* (f, 68), Challeng* (m, 67), Compete* $(\mathrm{m}, 64)$, and Principle* $(\mathrm{m}, 55)$. (Note that "*”, denotes a wildcard character or characters, e.g. Lead* includes lead, leader, leading, leadership, etc.)

\subsection{Gendered Words by School}

One of the questions that drove this project was whether gendered words encouraged or discouraged 
recruitment to engineering overall. We investigated whether universities differed in the degree that they utilized gendered language on their website and whether this would be correlated with differences in female enrolment. As Table 2 shows, most universities use more masculine words overall, with an average 27 per 10,000 words being masculine, compared to 17 per 10,000 words being feminine. This is consistent with previous research that has examined the occurrence of gendered words in job advertisements in male dominated fields [10].

Unique gendered words that were found at ten or more schools were exclusively masculine: Analy* (10 schools), Challeng* (15), Compet* (13), Individual* (10), Lead* (13), and Principle* (14). The most frequent feminine words (used at 9 schools each) were: Respon*, Support*, Together*, and Understand*.

Schools varied in the number of total words on their websites (which is not correlated to the number of disciplines, and is only weakly correlated to the size of the program), and showed a great deal of difference the frequency of gendered words (Table 2). Fourteen schools used more masculine than feminine words, and four schools (McGill, SFU, UPEI, and Waterloo) used more feminine words than masculine (in terms of total instances). Our dependent variable, the percentage of instances of feminine words per gendered words by school ranges from 0 to 61, with an average of 33 percent. Table 1 shows the results of the percent of women enrolled, the percent of tenured faculty that are women, and Table 2 shows the control variable, the total number of [gendered] words on each website.

Surprisingly, larger fractions of feminine words to total words on website are negatively correlated with the fraction of women enrolled in these universities $(-0.17, p$ $=0.007$ ) and larger fractions of masculine words is positively correlated with the proportion of women enrolled $(0.21, p<0.001)$, while no correlation was found between fraction of feminine words out of gendered words and female enrolment $(-0.005, p=0.90)$.

There were positive correlations between percentage of female tenure-line faculty and both fractions of masculine and feminine words by total words $(0.55$ and $0.31, \mathrm{p}<0.001)$.

The regression analysis reinforces this pattern and shows that for every increase in tenure line female faculty, we can expect a 2.4 unit increase in the fraction of feminine words $(\beta=2.4 \mathrm{p}<0.001)$. These results also indicate that increases in the fraction of enrolled female students will have a negative impact on the fraction of feminine words $(\beta=-0.062, p<0.001)$ when we control for the total words on the schools engineering website, and the total number of students enrolled in the program. These factors explain 25 percent of the variance in the fraction of gendered words $\left(\mathrm{R}^{2}=0.25, \mathrm{~F}=55.9, \mathrm{p}<\right.$ $0.001)$.

Table 2: Summary of gendered words by school

\begin{tabular}{|c|c|c|c|c|c|c|c|}
\hline University & $\begin{array}{l}\text { Total } \\
\text { Words on } \\
\text { Website }\end{array}$ & $\begin{array}{l}\text { No. of } \\
\text { Gendered } \\
\text { Words }\end{array}$ & $\begin{array}{l}\text { No. of } \\
\text { Fem. } \\
\text { Words }\end{array}$ & $\begin{array}{l}\text { No. of } \\
\text { Masc. } \\
\text { Words }\end{array}$ & $\begin{array}{l}\text { Percent } \\
\text { Feminine } \\
\text { Words }\end{array}$ & $\begin{array}{l}\text { Feminine Words } \\
\text { (per } 10,000 \text { Words } \\
\text { on Website) }\end{array}$ & $\begin{array}{l}\text { Masculine Words } \\
\text { (per } 10,000 \text { Words } \\
\text { on Website) }\end{array}$ \\
\hline Alberta & 15273 & 80 & 33 & 47 & $41 \%$ & 22 & 31 \\
\hline $\mathrm{BCIT}$ & 8341 & 15 & 4 & 11 & $27 \%$ & 5 & 13 \\
\hline Calgary & 3995 & 27 & 7 & 20 & $26 \%$ & 18 & 50 \\
\hline Carleton & 30888 & 46 & 15 & 31 & $33 \%$ & 5 & 10 \\
\hline Concordia & 7404 & 23 & 7 & 16 & $30 \%$ & 9 & 22 \\
\hline Conestoga & 1489 & 12 & 4 & 8 & $33 \%$ & 27 & 54 \\
\hline Dalhousie & 13454 & 67 & 19 & 48 & $28 \%$ & 14 & 36 \\
\hline Manitoba & 21055 & 58 & 23 & 35 & $40 \%$ & 11 & 17 \\
\hline McGill & 35457 & 101 & 57 & 44 & $56 \%$ & 16 & 13 \\
\hline Memorial & 4393 & 23 & 7 & 16 & $30 \%$ & 16 & 36 \\
\hline Regina & 2580 & 4 & 0 & 4 & $0 \%$ & 0 & 16 \\
\hline SFU & 5968 & 36 & 22 & 14 & $61 \%$ & 37 & 23 \\
\hline StFX & 864 & 1 & 0 & 1 & $0 \%$ & 0 & 12 \\
\hline UBCO & 2613 & 9 & 4 & 5 & $44 \%$ & 15 & 19 \\
\hline UBCV & 14531 & 40 & 1 & 39 & $3 \%$ & 1 & 27 \\
\hline UNB & 16732 & 44 & 11 & 33 & $25 \%$ & 7 & 20 \\
\hline UPEI & 350 & 5 & 3 & 2 & $60 \%$ & 86 & 57 \\
\hline Waterloo & 15742 & 75 & 39 & 36 & $52 \%$ & 25 & 23 \\
\hline Summary & $\begin{array}{l}\text { Total } \\
201,129 \\
\text { Mean } \\
11,174 \\
\end{array}$ & $\begin{array}{l}\text { Total } 666 \\
\text { Mean } 37\end{array}$ & $\begin{array}{l}\text { Total } \\
256 \\
\text { Mean } \\
14\end{array}$ & $\begin{array}{l}\text { Total } \\
410 \\
\text { Mean } \\
23\end{array}$ & Mean 33\% & $\begin{array}{l}\text { Mean } 17 \text { per } \\
10,000\end{array}$ & $\begin{array}{l}\text { Mean } 27 \text { per } \\
10,000\end{array}$ \\
\hline
\end{tabular}




\subsection{Gendered Words by Discipline}

Our next inquiry was whether there was variation in gendered wording across disciplines, as some disciplines are commonly understood to be more heavily male dominated than others (i.e. low female enrolment in Mechanical, high in Environmental). Enrolment numbers bear this out - the average proportion of women ranges between $8 \%$ for Computer Engineering and 37\% for Biosystems. There is a wider range of female enrolment by discipline than by school.

Because we are interested in how disciplinary cultures differ in their depiction of a strong masculine culture that might be perceived as unwelcoming to women, we restricted our analysis to specific disciplinary areas, and dropped general or common year observations.

As expected from the analysis by school, there are fewer female words when examined by discipline as well. There were 67 observed feminine words, and 149 observed masculine words (unique words: 16 masculine and 10 feminine). The most common gendered words used in discipline-specific material were Principle* $(\mathrm{m}, 10$ disciplines), Understand* (f, 10), Analy* (m, 9), Challeng* $(\mathrm{m}, 9)$, and Lead* $(\mathrm{m}, 8)$.

The number of women students in a discipline is not significantly correlated with the proportion of masculine or feminine words, but does have a weak, negative association with the fraction of feminine words of all gendered words that appear on discipline area web pages (Pearson's $r=-0.23, p<0.001$ ). Regression results mirror this finding, although because we have only limited discipline level data they are not reported here.

(While six of these schools have software engineering programs, the material from those programs contained no gendered words, therefore they are not included here.)

Table 3: Summary of gendered words by discipline

\begin{tabular}{|l|c|c|c|c|}
\hline Discipline & $\begin{array}{l}\text { Percent } \\
\text { Women } \\
\text { Students }\end{array}$ & $\begin{array}{l}\text { No. of } \\
\text { Fem. } \\
\text { Words }\end{array}$ & $\begin{array}{l}\text { No. of } \\
\text { Masc. } \\
\text { Words }\end{array}$ & $\begin{array}{l}\text { Percent } \\
\text { Feminine } \\
\text { Words }\end{array}$ \\
\hline Biosystems & $37 \%$ & 2 & 7 & $22 \%$ \\
\hline Chemistry & $29 \%$ & 5 & 12 & $29 \%$ \\
\hline Civil & $24 \%$ & 16 & 29 & $36 \%$ \\
\hline Computer & $8 \%$ & 9 & 15 & $38 \%$ \\
\hline Electrical & $14 \%$ & 14 & 16 & $47 \%$ \\
\hline $\begin{array}{l}\text { Engineering } \\
\text { Physics }\end{array}$ & $14 \%$ & 0 & 8 & $0 \%$ \\
\hline Environment & $32 \%$ & 1 & 11 & $8 \%$ \\
\hline Geology & $31 \%$ & 1 & 3 & $25 \%$ \\
\hline Industrial/ & $25 \%$ & 2 & 6 & $25 \%$ \\
Manufact. & $23 \%$ & 4 & 4 & $50 \%$ \\
\hline Materials & $12 \%$ & 8 & 32 & $20 \%$ \\
\hline Mechanical & $13 \%$ & 5 & 6 & $45 \%$ \\
\hline Mining & $25 \%$ & 10 & 16 & $38 \%$ \\
\hline Other & & $\begin{array}{l}\text { Mean } \\
\mathbf{6}\end{array}$ & $\begin{array}{l}\text { Mean } \\
\mathbf{1 3}\end{array}$ & $\begin{array}{l}\text { Mean } \\
\mathbf{3 0} \%\end{array}$ \\
\hline & Mean 22 & &
\end{tabular}

\section{DISCUSSION}

We found that masculine words predominated among gendered words in the materials collected, in total percentage of instances and in most commonly used words (overall and by number of schools).

\subsection{Summary of motivation and results}

The intention of this project was to help determine whether the public-facing materials that prospective students engage with may be a target for change when trying to recruit additional qualified women to engineering programs. If there is evidence of gendered wording in undergraduate engineering recruitment materials, then steps can be taken to include a balance of female-associated words in recruitment materials.

\subsection{Significance of findings}

We found that the fraction of female words to total words at an institution did not positively correlate with female enrolment; it appears that as percentage of female students increases, the percentage of feminine words decreases. It is not clear what the relationship between the two factors might be. Since we have only total enrolment data, rather than entrance data, we cannot account for the number of women students persisting in engineering at each school in relation to the number of women initially admitted. It may be the case that the schools with more feminine words project a more welcoming environment, and then women students arrive and find this is not the case; that is, a difference between the number of women initially recruited and the number of women who continue might exist, and may be indicative of factors such as school culture in combination with gendered recruitment materials. It may also be that as success in recruiting women increases, the perception of the need to cater toward female prospective students decreases.

Another interesting finding was that the proportion of tenure-line female faculty was associated with higher proportions of gendered words (both feminine and masculine), and regression results indicated that increasing female faculty led to increasing feminine words. Again, it is not clear how these factors are related. It may be possible that higher numbers of female faculty lead to more awareness of student gender imbalance, which leads to efforts to attract more female students, or simply that some influence of female faculty is seen in the public-facing materials, without having a concerted effort at female student recruitment. The literature shows that women's persistence in engineering and science is correlated with percentage of female faculty [12], 
however we did not find a significant correlation in this sample.

\subsection{Strengths and Limitations}

Strengths of this work include using a validated list of words that have been shown to affect perceptions of the climate and affinity for occupations in research subjects, as well as analyzing a wide range of Canadian engineering programs.

The gendered words list used in this study was created out of job advertisements, rather than undergraduate recruitment materials. While the occupations examined to produce the list included engineering, the list was not specific to undergraduate engineering recruitment.

The engineering school materials were collected during the 2014-2015 school year (and possibly revised to target the 2015-2016 incoming class), however enrolment data was only available from Engineers Canada for the 2013-2014 school year. It is likely that some recruitment materials changed between the time new students chose their program for 2013-2014 and the collection of materials, however it is unclear if these changes would include the type of wording we examined. We suspect engineering schools may not explicitly target wording changes at this level of subtlety. Future work may include re-assessing this data once the enrolment data for 2015-2016 becomes available. Additionally, we did not have access to data for only incoming or first year students, thus our enrolment data may be influenced by attrition as well as recruitment.

We limited our collection to English-language engineering schools because we were not confident that the gendered word list would translate into French and still contain the same underlying meaning experienced by English-speakers.

\subsection{Application of findings}

While several findings in this study about relationships between student gender, faculty gender, and gendered words were unexpected, we believe they lead to other interesting questions. This work provides a starting point for further exploration of the recruitment of women into undergraduate engineering, particularly through written materials.

The approach of using words that are associated with women to attract more female applicants has advantages over more explicit approaches (specific recruitment materials directed at women, for example). It can help avoid the results of "spotlighting," a type of bias where programs or materials are explicitly targeted to women (even in a positive way), and end up singling them out [13]. Further work creating our own recruitment materials using gendered words and testing them in populations of interest (high school students) may assist in determining the utility of this approach.

Future work could consider additional components of recruitment materials, including website images. Qualitative interviews or focus groups with women engineering students about their decision to pursue engineering could also yield valuable insight. Research with women who have chosen not to pursue engineering, despite an interest in the field, would also be fruitful although challenging to design given the elusiveness of this group.

\section{CONCLUSIONS}

Predominantly masculine gendered words were found in English-language Canadian engineering undergraduate recruitment materials. We found complex relationships between percentages of female students, female faculty, and gendered words. To our knowledge, this is the first evaluation of gendered wording in recruitment materials as a factor in the enrolment of women in engineering, and provides a foundation for future work in this area.

\section{Acknowledgements}

The authors would like to acknowledge Westcoast Women in Engineering, Science and Technology (WWEST) for providing funding, and to thank Diana Demmers for assistance in data preparation, and Rima Wilkes and Peter Ostafichuk for providing feedback on this work.

\section{References}

[1] Engineers Canada, "Canadian Engineers for Tomorrow: Trends in Engineering Enrolment and Degrees Awarded 2009-2013," 2014.

[2] Engineers Canada, "Strategies and Goals for Women in Engineering Activities," 2011.

[3] Association of Unversities of Canada, "Trends in Higher Education," Trends High. Educ., vol. 1, pp. 568, 2011.

[4] E. Smith, "Women into science and engineering? Gendered participation in higher education STEM subjects," Br. Educ. Res. J., vol. 37, no. 6, pp. 9931014, Dec. 2011.

C. J. Poor and S. Brown, "Increasing retention of women in engineering at WSU: A model for a women's mentoring program," Coll. Stud. J., vol. 47, no. 3, pp. 421-429, 2013.
C. De Cohen, "Retention is not the problem," Prism Mag. ASEE, no. OCTOBER, p. 55, 2009. 
[7] S. J. Ceci, W. M. Williams, and S. M. Barnett, "Women's Underrepresentation in Science: Sociocultural and Biological Considerations," Psychol. Bull., vol. 135, no. 2, pp. 218-261, 2009.

[8] “2014-06, 'National Graduates Survey, 2013 (Class of 2009-2010)', http://hdl.handle.net/11272/10040 UNF:5:gnm0E/9EyTbWxBNz6kWcJg== Statistics Canada [Distributor] V4 [Version].”.

[9] J. Acker, "Hierachies, Jobs, Bodies: A Theory of Gendered Organizations," Gend. Soc., vol. 4, no. 2, pp. 139-158, 1990.

[10] D. Gaucher, J. Friesen, and A. C. Kay, "Evidence that gendered wording in job advertisements exists and sustains gender inequality.," J. Pers. Soc. Psychol., vol. 101, no. 1, pp. 109-28, Jul. 2011.

[11] B. A. Nosek, M. Banaji, and A. G. Greenwald, "Harvesting implicit group attitudes and beliefs from a demonstration web site.," Gr. Dyn. Theory, Res. Pract., vol. 6, no. 1, pp. 101-115, 2002.

[12] G. Sonnert, M. F. Fox, and K. Adkins, "Undergraduate Women in Science and Engineering: Effects of Faculty, Fields, and Institutions Over Time," Soc. Sci. Q., vol. 88, no. 5, pp. 1333-1356, 2007.

[13] L. A. McLoughlin, "Spotlighting: Emergent Gender Bias in Undergraduate Engineering Education," J. Eng. Educ., vol. 94, no. 4, pp. 373-381, 2005.

\section{APPENDIX A: LIST OF GENDERED WORDS}

\section{A.1 List of male- and female-dominated occupations/fields used in gendered word list creation}

In Gaucher's original study, 11 gendered occupations were chosen to provide job advertisements from which gendered words were collected. From [10]: "Maledominated jobs were plumber ( $1 \%$ women), electrician $(2 \%)$, mechanic $(2 \%)$, engineer $(11 \%)$, security guard $(23 \%)$, and computer programmer $(26 \%)$; femaledominated jobs were administrative assistant (97\%), early childhood educator (94\%), registered nurse (90\%), bookkeeper $(90 \%)$, and human resources professional (71\%)." Additionally, co-op job advertisements for two faculties at the University of Waterloo were examined arts (female-dominated) and engineering (male dominated).

\section{A.2 Gendered words}

See Table 4 below for the gendered words list from the work of Gaucher et al. [10]. Note that the asterisk (*) indicates a wildcard, and any ending would be included (e.g. "child*" includes child, childhood, children, etc.).

Table 4: Gendered word list from Gaucher et al.

\begin{tabular}{|c|c|}
\hline MASCULINE & FEMININE \\
\hline Active & Affectionate \\
\hline Adventurous & Child $^{*}$ \\
\hline Aggress* & Cheer $^{*}$ \\
\hline Ambitio* & Commit* \\
\hline Analy* & Communal \\
\hline Assert* & Compassion* \\
\hline Athlet ${ }^{*}$ & Connect* $^{*}$ \\
\hline Autonom* & Considerate \\
\hline Boast $^{*}$ & Cooperat* $^{*}$ \\
\hline Challeng* $^{*}$ & Depend* $^{*}$ \\
\hline Compet $^{*}$ & Emotiona* \\
\hline Confident & Empath* \\
\hline Courag* & Feminine \\
\hline Decide & Flatterable \\
\hline Decisive & Gentle \\
\hline Decision* & Honest \\
\hline Determin* & Interdependen* \\
\hline Domina* & Interpersona* \\
\hline Force* & Kind \\
\hline Greedy & Kinship \\
\hline Headstrong & Loyal $^{*}$ \\
\hline Hierarch* & Modesty \\
\hline Hostil* & Nag \\
\hline Implusive & Nurtur* \\
\hline Independen* & Pleasant* \\
\hline Individual* $^{*}$ & Polite \\
\hline Intellect ${ }^{*}$ & Quiet* $^{*}$ \\
\hline Lead* $^{*}$ & Respon* \\
\hline Logic & Sensitiv* \\
\hline Masculine & Submissive \\
\hline Objective & Support $^{*}$ \\
\hline Opinion & Sympath* \\
\hline Outspoken & Tender* \\
\hline Persist & Together* \\
\hline Principle* $^{*}$ & Trust $^{*}$ \\
\hline Reckless & Understand $^{*}$ \\
\hline Stubborn & Warm* \\
\hline Superior & Whin* \\
\hline Self-confiden* & Yield* \\
\hline \multicolumn{2}{|l|}{ Self-sufficien* } \\
\hline Self-relian* & \\
\hline
\end{tabular}

